\title{
EL LABERINTO DE LA SOMATIZACIÓN: SE BUSCAN SALIDAS
}

\author{
JOSÉ LÓPEZ-SANTIAGO ${ }^{1}$ Y AMPARO BELLOCH ${ }^{2}$ \\ ${ }^{1}$ Unidad de Salud Mental, Complejo Hospitalario Universitario de Albacete, Albacete, España \\ ${ }^{2}$ Facultad de Psicología, Universidad de Valencia, Valencia, España
}

\begin{abstract}
Resumen: El trastorno de somatización (TS) fue introducido como síndrome en el DSM-III (1980) y, muy probablemente, desaparecerá en el DSM-V como categoría diagnóstica. En este trabajo se revisan las principales investigaciones que ha generado el estudio del TS en los últimos 30 años respecto a su epidemiología, comorbilidad, y variables psicológicas con las que se ha asociado: preocupación por la enfermedad, amplificación somato-sensorial, atribución somática, alexitimia y neuroticismo. También se analizan las diferentes propuestas nosológicas surgidas en la pasada década y el debate suscitado hasta llegar a la actual propuesta de clasificación en el DSM-V. Se considera necesaria y positiva la radical modificación que propone el grupo de trabajo del DSM-V, aunque también se exponen los riesgos de esta propuesta en dos aspectos cruciales: agrupar dentro del mismo diagnóstico los actuales TS e hipocondría, e incluir en la misma categoría síntomas no explicados y facticios.
\end{abstract}

Palabras clave: Trastorno de somatización; trastornos somatoformes; hipocondría; síntomas somáticos; DSM-V.

The somatization labyrinth: «Way out wanted»

\begin{abstract}
Somatization disorder (SD) was included as a new syndrome in the DSM-III (APA, 1980), but its removal from the DSM-V as a diagnostic category is more than probable. This work examines the main studies that SD has generated in the last 30 years as regards epidemiology, comorbidity, and associated psychological variables: illness concern, somatosensory amplification, somatic attribution, alexithymia, and neuroticism. The several nosological proposals in the past decade are also revised, as well as the debate that developed until the current DSM-V classification proposals. The radical changes proposed by the DSM-V work group are considered necessary and positive, although two risks of the proposal are also exposed: clustering under the same diagnostic category the current $\mathrm{SD}$ and hypochondriasis, and including non-explained and factitious symptoms in the same category.
\end{abstract}

Keywords: Somatization disorder; somatoform disorders; hypochondriasis; somatic symptoms; DSM-V.

\section{INTRODUCCIÓN}

Experimentar síntomas somáticos es un fenómeno común a casi cualquier situación vital humana, muy especialmente cuando la experiencia conlleva sufrimiento. La universalidad del fenómeno de la somatización se refleja en muchos estudios en los que se constata, por ejemplo, que el $80 \%$ de las personas presenta algún sínto-

Recibido: 2 febrero 2012; 15 marzo 2012.

Correspondencia: José López-Santiago, Hospital Perpetuo

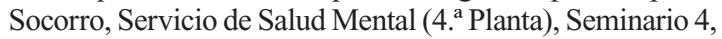
02006 Albacete, España. Correo-e: jlopezs@sescam.jccm.es ma somático a lo largo de una semana (p.ej., Kellner, 1985), o que el 30\% de las consultas en atención primaria se deben a síntomas no explicados médicamente (p.ej., Kroenke, 2003). No obstante, resulta llamativo que resultados como éstos se inviertan cuando lo que se analiza es la categoría diagnóstica de trastorno de somatización (TS), pues los estudios epidemiológicos suelen cifrar su prevalencia alrededor del $0,4 \%$, tasa que llega a ser solo del $0,03 \%$ en otros casos (p.ej., Creed y Barsky, 2004). Estos datos revelan una presencia casi residual del TS, al menos tal y como se define desde la tercera edición del Diagnostic and Statistical Manual of Mental 
Disorders, DSM-III (APA, 1980). En los 30 años transcurridos desde la «oficialidad» del TS en este manual, se ha oscilado entre dos extremos: por un lado, la inespecificidad de la experiencia sintomática de la somatización, y por el otro la excesivamente específica y poco operativa definición, según la mayoría de autores, de la categoría nosológica del TS. En el camino recorrido desde entonces se han producido muchos intentos por encontrar una vía intermedia, pero éstos casi siempre han interesado solo al círculo restringido de investigadores preocupados por el TS con el consiguiente escaso impacto en la comunidad científica general. Por ese motivo, los cambios presuntamente significativos en la categoría diagnóstica que finalmente englobe el fenómeno de la somatización que se anuncian para las próximas ediciones del DSM-V y la International Classification of Diseases (CIE-11, OMS), van a tener una repercusión amplia en el futuro de este problema, tanto en lo que respecta a su investigación como en cuanto a su comprensión y abordaje clínicos. Una muestra mínima pero elocuente de ello es la muy probable sustitución del mismo término somatización. El objetivo de este artículo es, primero, analizar la literatura científica acumulada en las últimas décadas sobre la delimitación diagnóstica y las características clínicas propias de los pacientes que son diagnosticados de TS, para a continuación realizar una valoración crítica de los criterios que se están proponiendo para su diagnóstico.

\section{NOSOLOGÍA DEL TRASTORNO DE SOMATIZACIÓN}

La caracterización actual del TS tiene como antecedente más remoto el concepto de histeria $\mathrm{y}$ en concreto el trastorno denominado histeria o sindrome de Briquet (Guze, Woodruff y Clayton, 1972), entidad diagnóstica establecida apartir de los estudios del grupo de San Luis de la Universidad de Washington (Guze, 1963, 1967; Perley y Guze, 1962) con pacientes mujeres polisintomáticas. El cuadro presentaba elevada fiabilidad (Spitzer, Endicott y Robins, 1978), adecuada validez (Guze, 1970) y era estable en el tiempo (Perley y Guze, 1962). Además, se trataba de un trastorno relativamente común, con una prevalencia de entre $1-2 \%$ en mujeres (Woodruff, Clayton y Guze, 1971) y un patrón familiar que afectaba a entre el $10 \%$ y el $20 \%$ de familiares de primer grado (Cloninger, Reich y Guze, 1975). Este significativo apoyo científico facilitó que la definición que el grupo de San Luis planteaba sobre la histeria fuera incorporada a los criterios de Feighner et al. (1972), en los Research Diagnostic Criteria (Spitzer, Endicott y Robins, 1975) y, finalmente, en el DSM-III y en la CIE10 (OMS, 1992) con la denominación de tratorno de somatización. En la Tabla 1 se exponen sintéticamente los hitos más significativos en torno a la evolución del TS y las propuestas de futuro.

Tabla 1. Hitos significativos en la evolución histórica del trastorno de somatización

\begin{tabular}{|c|c|c|}
\hline Fecha & Autor(es) & Hito \\
\hline 1859 & Briquet & Traité clinique et thérapeutique de l'hystérie \\
\hline 1909 & Savill & Criterios diagnósticos de la histeria \\
\hline 1951 & Purtell, Cohen y Robins & $\begin{array}{l}\text { Observaciones de aspectos clínicos de la histeria: estudio cuantita- } \\
\text { tivo de } 50 \text { pacientes }\end{array}$ \\
\hline $1962-1963$ & Perley y Guze & $\begin{array}{l}\text { Histeria. Estabilidad y utilidad de criterios clínicos.Observaciones } \\
\text { respecto a la historia natural de la histeria. }\end{array}$ \\
\hline 1972 & $\begin{array}{l}\text { Feighner, Robins, Guze, Woodruff, } \\
\text { Winokur, Muñor }\end{array}$ & Criterios diagnósticos para su uso en investigación psiquiátrica. \\
\hline $1975 / 1985$ & Spitzer, Endicott y Robins & Criterios diagnósticos de investigación (RDC) \\
\hline 1980 & APA/DSM-III & Trastornos somatoformes. Trastorno de somatización. \\
\hline 1986 & APA/DSM-III-R & Trastorno somatoforme indiferenciado \\
\hline 1992 & OMS/CIE-10 & Trastornos somatoformes \\
\hline 2010 & APA-Grupo de Trabajo DSM-V & $\begin{array}{l}\text { Propuestas: a) Eliminación categoría de trastornos somatoformes; } \\
\text { b) Nuevo: Trastorno de síntomas somáticos }\end{array}$ \\
\hline
\end{tabular}


El TS es el principal trastorno organizador del grupo de los trastornos somatoformes, introducido como ya se ha dicho en la tercera edición del DSM, para describir una nueva categoría de trastornos mentales cuya característica común es la presencia de "síntomas físicos que sugieren una alteración somática para la que no existen hallazgos orgánicos demostrables o mecanismos fisiológicos conocidos y en los que hay pruebas positivas o presunciones firmes de que los síntomas se encuentran ligados a factores o conflictos psicológicos» (APA, 1980; p. 305). Posteriormente, en la versión revisada (DSM-III-R; APA, 1987) se incluyeron siete trastornos bajo la categoría de trastornos somatoformes, que se mantuvieron en el DSM-IV (1994): TS, somatoforme indiferenciado, de conversión, dolor somatoforme (trastorno de dolor, en el DSM-IV), hipocondría, dismórfico, y somatoforme no especificado. Respecto a la clasificación de la OMS, los trastornos somatoformes se incluyeron en la CIE10 (OMS, 1992) dentro de la categoría Trastornos neuróticos, secundarios a situaciones estresantes, y somatoformes. La divergencia más notable entre la CIE-10 y el DSM-IV se centra en el hecho de que en la CIE el trastorno de conversión no se incluye entre los trastornos somatoformes, sino en los disociativos (de conversión).

En cuanto a las características diagnósticas que se incluyen en las clasificaciones, algunas resultan controvertidas o han mostrado considerable inconsistencia. Ello ha llevado al consenso entre los expertos sobre la necesidad de modificar sustancialmente los actuales criterios. Entre los aspectos más controvertidos destacan los cinco siguientes (López-Santiago, 2011; López-Santiago y Belloch, 2002): (a) El número de síntomas requeridos para el diagnóstico, que ha pasado de 25 de la definición original a los 8 actuales del DSM-IV, frente a la ausencia de un número específico en la CIE-10. (b) Los síntomas considerados como somatizaciones, que no son equivalentes en las distintas clasificaciones ni en los instrumentos de evaluación. (c) Falta de acuerdo sobre si los síntomas constituyen una categoría general o deben evaluarse sobre la base de diferentes sistemas orgánicos, como se propone en el DSM-IV. (d) Debate sobre si debe tenerse en cuenta el mismo número de síntomas para hombres y mujeres. (e) Falta de acuerdo sobre si rechazar los resultados que desconfirman una enfermedad médica conocida, es un rasgo definitorio del trastorno, como propone la CIE-10, o si el diagnóstico debe hacerse sobre la base de síntomas actuales o a lo largo de la vida.

\section{EPIDEMIOLOGÍA}

El estudio de la epidemiología del TS resulta especialmente complejo debido a la inconsistencia que suelen presentar los resultados de los estudios y porque habitualmente en su análisis se mezclan tres aproximaciones distintas al problema: (1) el estudio del trastorno de somatización como categoría nosológica (DSM o CIE); (2) la utilización de definiciones alternativas y menos restrictivas del fenómeno de la somatización, como el constructo de somatización abreviada (Escobar et al., 1987); y (3) el estudio de la elevada presencia de somatización en las consultas médicas. Otro problema se refiere a las dificultades del propio proceso de evaluación, debido a los diferentes criterios diagnósticos de somatización utilizados, a las diferencias en los instrumentos de evaluación, al modo en que se realiza la entrevista y quién la realiza, a los diferentes formatos de respuesta (listado abierto o cerrado de síntomas), y a si se hace referencia a síntomas actuales o a síntomas a lo largo de la vida (Escobar y Gureje, 2007).

Tradicionalmente se ha considerado que la presencia de síntomas somáticos constituye un fenómeno extremadamente común en la población general, además de suponer un porcentaje muy elevado de las quejas manifestadas por los pacientes en las consultas médicas (Kellner, 1985). Schappert (1992) concluye que estos síntomas constituyen el 50\% de las consultas médicas ambulatorias, lo que supone la estimación de aproximadamente 400 millones de consultas médicas anuales en Estados Unidos.

A pesar de la unanimidad a la hora de concluir que la presencia de síntomas somáticos no explicados es un fenómeno muy común, existe 
una enorme variabilidad para informar de la prevalencia de este fenómeno, desde estudios que cifran el fenómeno de la somatización en un $10 \%$ de la población general (Kirmayer, 2004) hasta estudios que encuentran que más de un $70 \%$ de los síntomas referidos no tienen una explicación médica (Kroenke, Arrington y Mangesdorff, 1990). La mayoría de las revisiones sitúan este fenómeno entre un 20\% y 30\% (Kroenke, 2003; Kirmayer, Groleau, Looper y Dao, 2004). Dada la heterogeneidad de los datos resulta difícil precisar cuál es su verdadera frecuencia. Por ejemplo, en muestras representativas de más de 2.500 sujetos, en función de la metodología utilizada a la hora de recoger la información, se ha informado en algunos casos que el $10,5 \%$ de la población refiere algún síntoma de este tipo (Kirmayer et al., 2004) mientras que en otros estudios la cifra asciende al 81,6\% (Hiller, Rief y Brahler, 2006).

En lo que sí parecen ser consistentes los resultados de las investigaciones es en los sínto- mas más habituales, que suelen coincidir con los enumerados por el Patient Health Questionnaire (PHQ-15; Kroenke, Spitzer, Williams, 2002) y que se muestran en la Tabla 2. Probablemente Kroenke $(2003,2006,2008)$ es el autor que de forma más sistemática ha estudiado en las últimas dos décadas el problema de los síntomas físicos en la población general y en las consultas médicas. Sus principales conclusiones se pueden resumir como sigue: primero, en torno al $80 \%$ de la población general experimenta algún síntoma somático en el periodo de un mes, si bien la mayoría de estos síntomas son autolimitados pues solo se consulta por un $25 \%$ de los mismos. En segundo lugar, las variables más determinantes en relación con la búsqueda de ayuda sanitaria por causa de los síntomas son las expectativas y preocupaciones negativas sobre ellos. Y, tercero, en torno al $75 \%$ de los síntomas se resuelven sin intervención en el plazo de pocas semanas, mientras que el restante $20-25 \%$ se mantienen durante un tiempo mayor.

Tabla 2.Principales síntomas identificados como somatizaciones

\begin{tabular}{ll}
\hline \multicolumn{1}{c}{$\begin{array}{c}\text { Síntomas más frecuentes } \\
\text { (Rief et al., 2001; Hiller et al., 2006) }\end{array}$} & \multicolumn{1}{c}{$\begin{array}{c}\text { Síntomas en la escala PHQ-15 } \\
\text { (Kroenke, 2008) }\end{array}$} \\
\hline $\begin{array}{l}\text { 1. Dolor de cabeza } \\
\text { 2. Dolor abdominal }\end{array}$ & $\begin{array}{l}\text { 1. Dolor de estómago } \\
\text { 3. Dolor de espalda }\end{array}$ \\
$\begin{array}{l}\text { 2. Dolor lumbar } \\
\text { 4. Dolor en las articulaciones }\end{array}$ & 3. Dolor en brazos piernas o articulaciones \\
5. Dolor en piernas y/o brazos & 5. Dolor de cabeza \\
6. Pesadez/hinchazón & 6. Dolor torácico \\
7. Regurgitación de comida & 7. Mareos \\
8. Intolerancia a alimentos & 8. Desvanecimientos \\
9. Mal sabor de boca/lengua pastosa & 9. Sentir que el corazón palpita o se acelera \\
10. Boca seca & 10. Dificultad para respirar \\
10. Frecuencia urinaria & 11. Dolor / problemas durante relaciones sexuales \\
12. Palpitación & 12. Estreñimiento, heces sueltas o diarreas \\
13. Malestar estómago, estómago revuelto & 13. Nauseas, gases, indigestión \\
14. Sudoración (fría o caliente) & 14. Sentirse cansado o tener baja energía \\
15. Excesivo cansancio tras ejercicio leve & 15. Problemas para dormir \\
16. Indiferencia sexual &
\end{tabular}

Nota. PHQ-15 = Patient Health Questionnaire 


\section{Prevalencia del Trastorno de somatización}

Uno de los principales problemas que tiene la caracterización actual del TS es su escasa representación en los estudios epidemiológicos que han examinado su prevalencia a partir de criterios diagnósticos DSM o CIE. Este aspecto resulta especialmente paradójico teniendo en cuenta que el fenómeno clínico de la somatización es habitualmente referido como muy frecuente. Por ejemplo, los trastornos somatoformes se suelen incluir en el grupo de los más frecuentes en las consultas de atención primaria, con tasas muy similares a las de los trastornos de ansiedad y depresivos (Spitzer et al., 1994). Incluso algunos estudios epidemiológicos en atención primaria sitúan estos trastornos como tres veces más frecuentes que los de ansiedad y del estado de ánimo ofreciendo datos superiores en algunos casos al 20\% (Hanel et al., 2009; Toft, et al., 2005; De Waal et al., 2008). Como es natural, si estos datos se ajustan a la realidad, el coste económico y el consumo de recursos sanitarios generados por estos trastornos son muy elevados (Barsky, Orav y Bates, 2005).

Como no puede ser de otro modo, la mayoría de casos identificados en los estudios mencionados se deben a diagnósticos de trastorno somatoforme indiferenciado (TSI), definido por la presencia de al menos un síntoma físico no explicado médicamente con duración superior a 6 meses. La prevalencia de este diagnóstico en los estudios poblacionales está estimada en el 13,4\% (Faravelli el al., 1997), incrementándose como ya se ha dicho hasta el $20 \%$ en atención primaria. No obstante, hay que recordar aquí que el TSI no se ha considerado hasta la fecha como una entidad clínicamente validada (Mayou et al., 2005).

Por lo que se refiere estrictamente al TS, los resultados del Epidemiologic Catchment Area Program (ECA), desarrollado en la década de 1980 en Estados Unidos (Robins y Regier, 1991) sobre una población total cercana a las 20.000 personas, indicaban ya prevalencias muy bajas: del 0,12\% (Robins et al., 1984), del 0,03 (Escobar et al., 1987) y del 0,38\% (Swartz, Blazer, George y Landerman, 1986), con una media del 0,13\% (Kirmayer y Tailleifer, 1996). En el estudio epidemiológico de Gureje et al.
(1997) realizado bajo los auspicios de la OMS en 14 países, se indicaba una prevalencia del TS del 2.8\% según criterios diagnósticos CIE-10, si bien se detectaba también gran variabilidad entre los países participantes en el estudio (rango de $17,7 \%$ a $0,1 \%$ ). Cuando se toman los criterios DSM la prevalencia es del 1,4\% (Gureje et al, 1997, Simon y Gureje, 1999). Resultados parecidos los ofrecieron Fink et al. (1999), que encontraron una prevalencia del TS en atención primaria del 5,7\% según criterios CIE-10 y del $0,8 \%$ con criterios DSM-IV. Finalmente, las principales revisiones más recientes sobre la epidemiología del TS en población general ofrecen resultados inferiores al 1\% (Creed y Barsky, 2004; Hiller et al., 2006). A partir de estos datos, muchos autores han cuestionado la representatividad y utilidad de la categoría actual de TS (Kroenke, Sharpe y Sykes, 2007), ya que parece representar un extremo del espectro del fenómeno clínico de la somatización. Y aunque también es cierto que algunos estudios han informado de prevalencias mucho más elevadas (p.ej., Tolf et al., 2005), la verdad es que se trata más de una excepción a la norma general.

\section{Prevalencia del constructo de somatización abreviada}

Debido a las bajas tasas de prevalencia que acabamos de comentar y su no coincidencia con la impresión clínica respecto a la elevada frecuencia del fenómeno de la somatización, Escobar et al. (1987) elaboraron el constructo de somatización abreviada consistente en la presencia de 4 síntomas en hombres y 6 en mujeres. Esta definición ha tenido una amplia repercusión en la literatura, utilizándose por muchos autores como criterio diagnóstico válido en la clínica de TS. Creed y Barsky (2004) resumen los datos de la somatización abreviada y encuentran que en población general la prevalencia encontrada oscila entre el $4,4 \%$ y el $19 \%$, y en pacientes consecutivos atendidos en atención primaria la media es del 16,6\% (rango: 7,3\% $35 \%$ ). En población española, Lobo et al. (1996) encuentran un prevalencia del 7,3\%.

Respecto a variables sociodemográficas, el TS, al igual que el concepto de histeria, se ha 
relacionado tradicionalmente de forma exclusiva con el sexo femenino. Los datos de los que disponemos actualmente, obtenidos a partir de estudios epidemiológicos, aunque también sugieren una mayor prevalencia en mujeres indican a la vez que el TS no es tan exclusivo del sexo femenino como se presuponía, y que se aproxima a las ratios hombre/mujer de otros trastornos como ansiedad o depresión. Por ejemplo, en el trabajo multicéntrico de Gureje et al. (1997), aunque la presencia del trastorno es significativamente mayor en mujeres que en hombres (una prevalencia del 3,3\% en mujeres frente al 1,9\%), las diferencias ya no son tan extremas como habitualmente se ha supuesto. Con todo, los resultados son divergentes en función del concepto de somatización que se evalúe. Por lo que se refiere al constructo de somatización abreviada, la revisión de Creed y Barsky (2004) concluye que, de 14 estudios analizados, en 8 se encontró asociación con sexo femenino. La relación por sexos más habitual en este cuadro es $2: 1$. En relación a otras características sociodemográficas, la revisión de Creed y Barsky (2004) muestra que la asociación más consistente es con el bajo nivel de instrucción.

\section{VARIABLES EXPLICATIVAS DEL TRASTORNO DE SOMATIZACIÓN}

Más allá de los criterios de los manuales de diagnóstico psiquiátrico al uso, es importante realizar una aproximación más cercana a la clínica que presentan las personas con un TS. El objetivo de este apartado es presentar la información que estimamos más relevante sobre las investigaciones y/o explicaciones que en la actualidad intentar ofrecer información sobre las posibles causas del TS desde una perspectiva psicológica.

\section{Preocupación por la enfermedad y somatización}

La preocupación por la enfermedad es la característica en la que se ha basado sobre todo la definición de hipocondría (APA, 2000). Así, mientras que en la hipocondría la preocupación se ha asociado principalmente a la existencia de una enfermedad grave, en el TS la preocupación se ha asociado con el malestar y disfunción que directamente ocasionan los síntomas (Noyes, Stuart, Watson y Langbehn, 2006). A pesar de esta diferenciación, para algunos autores somatización e hipocondría pueden considerarse como parte de un mismo proceso (Kellner, 1990). Las puntuaciones elevadas en hipocondría se han relacionado con síntomas somáticos tanto en población general como en muestras clínicas. La mayoría de los estudios que establecen esta relación han sido realizados con el índice Whiteley (Noyes et al., 2006).

Sin embargo, sorprende la escasa presencia de estudios publicados que comparen directamente hipocondría con TS. Es posible que esta escasez de deba, en parte, a la norma sobre el diagnóstico jerárquico de TS respecto a hipocondría que plantea el DSM, puesto que impide contemplar la simultaneidad o co-ocurrencia de ambos diagnósticos en una misma persona. Esto podría explicar porqué algunas publicaciones bastante exhaustivas respecto a la comorbilidad del TS (p.ej., Brown, Golding y Smith, 1990) no ofrecen resultados de co-ocurrencia entre TS e hipocondría. A pesar de ello, según una revisión reciente (Noyes et al., 2006), la comorbilidad del TS en pacientes hipocondriacos (criterios DSM) era relativamente baja, entre el 7\% y el $21 \%$, mientras que cuando se utilizaban los criterios abreviados de Escobar (4 síntomas hombres/ 6 mujeres), la comorbilidad aumentaba sustancialmente, pues oscilaba entre el $32 \%$ y el $83 \%$. En una revisión anterior (Creed y Barsky, 2004), la comorbilidad hipocondría-TS oscilaba entre el $8 \%$ y el $60 \%$ en función de los criterios utilizados para definir el TS, de tal manera que la tasa aumenta sustancialmente cuanto menos restrictivos son los criterios para el diagnóstico del TS. Según Escobar et al. (1998), la prevalencia de hipocondría en pacientes somatizadores $(20 \%)$ era significativamente más alta que la prevalencia en pacientes no somatizadores $(3,1 \%)$. Por otra parte, en pacientes hipocondríacos la prevalencia del TS era del $8,2 \%$ frente al $1,1 \%$ en los no hipocondríacos. Otros estudios posteriores, como el de Leibbrand et al. (2000), informan de resultados similares. 


\section{Atribución de síntomas}

Desde diferentes perspectivas se han identificado tres dimensiones de la atribución de síntomas relacionadas con la conducta de enfermedad: (1) a factores psicológicos (2) a factores somáticos o de enfermedad física, y (3) a factores normales o ambientales (Robbins y Kirmayer, 1991). La atribución de los síntomas a causas normales o ambientales parece ser la característica más común tanto en la población no clínica (Kessler, Lloyd, Lewis y Gray, 1999), como en la que acude a atención primaria (Sensky, 1994). Por otra parte, diversos estudios han sido consistentes en mostrar que los pacientes aquejados de problemas psicológicos realizan más frecuentemente atribuciones de tipo psicológico (Kirmayer y Robbins, 1996; MacLeod, McNamee, Boyle, Offord y Friedrich, 1999). Finalmente, la atribución somática parece ser una característica relacionada con preocupaciones hipocondríacas, tanto en atención primaria (MacLeod et al.,1998), como en salud mental (Castañeiras, 2001; Henningsen, Jakobsen, Schiltenwolf y Weiss, 2005).

Por otra parte, la atribución de los síntomas influye en factores relacionados con la evolución y diagnóstico del trastorno. Kessler et al. (1999) han estudiado la relación entre estilo atribucional de síntomas evaluado con el Cuestionario de Interpretación de Síntomas (SIQ) y el reconocimiento por médicos de atención primaria de pacientes con elevados niveles de ansiedad y depresión. Cuando el estilo atribucional era normalizador, los médicos eran menos hábiles en identificar los cuadros de ansiedad. Por el contrario, cuando el estilo era preferentemente de origen psicológico, los médicos identificaban con mucha más frecuencia los síntomas mentales. Por otro lado, Parker y Parker (2003) encuentran, en atención primaria, que los pacientes depresivos que realizan una atribución psicológica de sus síntomas somáticos, etiquetan su depresión más frecuentemente como un «trastorno» y es más probable que reciban tratamiento para la depresión. Finalmente, desde otra perspectiva más cualitativa, Kirmayer et al. (2004) han estudiado la atribución de los síntomas a partir de la narrativa de los pacientes de su enfermedad: "¿Qué expli- cación se da la gente a sí misma respecto a sus síntomas?». Los autores encuentran que ninguna narrativa es mejor que otra. Sin embargo, las personas que no podían dar sentido a sus síntomas en ninguna dirección eran los que presentaban un mayor malestar y las que referían menor beneficio del tratamiento.

\section{Amplificación somatosensorial}

La amplificación de las sensaciones físicas como característica relacionada con diversos trastornos, tanto mentales como físicos, ha sido estudiada principalmente por Barsky y colaboradores durante las tres últimas décadas. La amplificación somatosensorial se define como la tendencia a experimentar las sensaciones somáticas como intensas, nocivas, y perturbadoras (Barsky, Goodson, Lane y Cleary, 1988). Aunque la amplificación somatosensorial se ha vinculado especialmente con la hipocondría (Barsky, Wyshak y Klerman, 1990; Belloch, Ayllón, Martínez, Castañeiras y Jiménez, 1999), los autores han relacionado el constructo con una variedad de condiciones clínicas, entre las que se encuentra la somatización (Barsky y Wyshak (1990). Estudios más recientes han mostrado una estrecha relación entre somatización y amplificación somatosensorial (Duddu, Isaac y Chaturdevi, 2006).

\section{Alexitimia y somatización}

El término alexitimia fue introducido en la literatura científica por Sifneos (1973) para definir a los «pacientes psicosomáticos». El concepto de alexitimia puede resumirse en la incapacidad para expresar el mundo emocional interno ya sea mediante palabras, ensueños o fantasías. Aunque se han desarrollado varios instrumentos para su evaluación el más utilizado y validado es la Escala de Alexitimia de Toronto (TAS) (Taylor, Ryan y Bagby, 1985), y más concretamente su versión reducida de 20 ítems (TAS-20; Bagby, Parker y Taylor, 1994), que se agrupan en 3 conjuntos o subescalas: (1) dificultad para identificar sentimientos y distinguir entre sentimientos y sensaciones corpora- 
les; (2) dificultad para describir sentimientos; $y$ (3) pensamiento orientado hacia lo exterior. En el primer estudio realizado en nuestro país sobre la relación entre alexitimia y síntomatología somática, Sandín, Santed, Chorot y Valiente (1996) constataron una estrecha relación entre el nivel de síntomas somáticos y la dimensión de alexitimia definida como dificultad para identificar y distinguir entre sentimientos y sensaciones corporales, asociación que no se daba para el resto de las dimensiones de alexitimia.

De Gucht (2003) realizó una revisión exhaustiva de la literatura respecto a la relación entre alexitimia y somatización y concluye que la mayoría de los estudios constatan una relación positiva entre ambas variables. De las tres dimensiones de la TAS es la dificultad para identificar sentimientos la que presenta una mayor asociación con somatización. Estudios posteriores a esta revisión confirman estos resultados (Pedrosa Gil et al., 2008). Respecto a estudios que han comparado sujetos con diagnósticos somatoformes, aunque en algunos se constata un mayor grado de alexitimia en sujetos somatoformes frente a grupos control o sujetos con enfermedad médica (Bach y Bach, 1996), cuando se comparan las personas con diagnóstico de somatización con población clínica de ansiedad, hipocondría o depresión las diferencias no son significativas (Duddu, Isaac y Chaturvedi 2003).

\section{Relación de somatización con depresión $y$ ansiedad}

Habitualmente se ha asumido que existe una estrecha asociación entre somatización y depresión (Katon, Kleinman y Rosen, 1982; Lipowski, 1990). Se ha encontrado consistentemente una asociación positiva entre somatización, por un lado y ánimo depresivo y ansiedad por otro, tanto en población general (Simon y VonKorff, 1991), como en atención primaria (Escobar et al., 1998). Estos resultados han sido confirmados recientemente en las investigaciones del grupo de Löwe y Henningsen (p. ej., Hanel, et al., 2009; Löwe, Spitzer et al., 2008).

Por otra parte, desde un enfoque dimensional, varios estudios han mostrado que cuando el número de síntomas somáticos se incrementa, también lo hacen los niveles de ansiedad y depresión (Katon et al., 1991; Kroenke, Spitzer, DeGruy y Swindl, 1998). Además cuando los síntomas de somatización aparecen asociados a trastornos de ansiedad y/o depresión, se agrava significativamente el cuadro clínico (Allen et al., 2001). Respecto a la dirección de la asociación, no existe evidencia que indique si la existencia de trastornos somatoformes primarios predice posteriores trastornos de ansiedad o depresivos, o viceversa (Lieb et al., 2007).

\section{Personalidad y somatización}

El TS se ha asociado con la personalidad en dos sentidos. En primer lugar, diversas investigaciones mostraron una elevada comorbilidad entre el TS, especialmente bajo el constructo histeria o síndrome de Briquet, y un trastorno de personalidad, en concreto la sociopatía, equivalente al actual trastorno de personalidad antisocial, en hombres, y con el trastorno histriónico en mujeres (Woodruff, Goodwin y Guze, 1978). No obstante, estudios más recientes, aunque evidencian elevadas tasas de comorbilidad con trastornos de personalidad, no han reflejado esa estrecha asociación con los trastornos de personalidad histriónico y antisocial (Kirmayer, Robbins y Paris, 1994; Noyes et al., 2006). Por otra parte, aunque son pocos los estudios que han evaluado dimensiones de personalidad en sujetos con trastorno de somatización, gran parte de los que han relacionado somatización con dimensiones de personalidad lo han hecho con neuroticismo (Deary, Chalder y Sharpe, 2007).

\section{EL LABERINTO SOMATOFORME: CONTROVERSIA ACTUAL Y PERSPECTIVAS DE FUTURO}

\section{«Abolición» de la categoría de trastornos somatoformes}

Tras el anuncio de la preparación de la $5 .^{\mathrm{a}}$ edición DSM (Kupfer, First y Rieger 2002), se ha iniciado en los últimos años un intenso de- 
bate respecto a la validez, fiabilidad y utilidad de la clasificación de los trastornos somatoformes. Mayou, Levenson y Sharpe (2003) publicaron en Psychosomatics, revista oficial de la Academy of Psychosomatic Medicine de Estados Unidos, una importante editorial en la que proponen una modificación radical de esta categoría, y abogan por su «abolición» y la reubicación de los trastornos que actualmente la componen. Podemos etiquetar la editorial de Mayou et al. como el motor del intenso y radical debate que en los últimos años se está produciendo en torno a los trastornos somatoformes.

Estas propuestas fueron refrendadas posteriormente por los mismos autores, junto con otros autores relevantes de la psiquiatría psicosomática norteamericana (Mayou, Kirmayer, Simon, Kroenke y Sharpe, 2005). En este trabajo, más elaborado y sistemático que el precedente, insisten, no obstante, en el carácter especulativo de la categoría cuando fue introducida en el DSM-III y las dificultades que con ésta surgen en la clínica, donde a pesar de tratarse de una clasificación de trastornos mentales, la mayoría de los pacientes con quejas somáticas no explicadas son atendidos por otros especialistas médicos distintos a los especialistas en salud mental. Los autores consideran que la actual categoría de trastornos somatoformes como entidad diagnóstica presenta múltiples defectos, que resumimos en la Tabla 3.

Tabla 3. Críticas a la categoría de trastornos somatoformes (Mayou et al., 2005)

1. El término es inaceptable para los pacientes. Remite al antiguo uso del término somatización y puede llevar a la negación del sufrimiento y realidad de los síntomas.

2. La categoría es inherentemente dualista. Es cuestionable la idea de que los síntomas somáticos pueden dividirse en síntomas que reflejan enfermedad y otros que tienen origen psicógeno.

3. No forman una categoría coherente. El solapamiento con muchos otros trastornos mentales, definidos en parte por síntomas físicos (como ansiedad y depresión), es una causa potencial de error diagnóstico.

4. Es incompatible con culturas no occidentales. Por ejemplo, la adaptación China del DSM excluye estos trastornos.

5. Es ambiguo en los criterios de exclusión con otros trastornos. Un paciente puede ser clasificado por el mismo problema tanto en el Eje I (p.ej., como trastorno somatoforme indiferenciado) como en el Eje III (p.ej., como colon irritable).

6. Muchas de las subcategorias no poseen fiabilidad.

7. Carece de clara definición del umbral de cuándo debe ser diagnosticado un trastorno somatoforme.

8. La ambigüedad de la categoría ocasiona problemas y disputas. Causa confusión y disputas en medicina legal y con las entidades de seguros.

Como corolario los autores proponen los siguientes cambios: en primer lugar, abolir la categoría de trastornos somatoformes del Eje I del DSM; en segundo término, reubicar algunas de las subcategorías, como por ejemplo, incluir hipocondría junto con los trastornos de ansiedad, y/o ubicar los trastornos conversivos y disociativos en un mismo grupo. Y, tercero, proponen considerar el continuo TS-trastorno somatoforme indiferenciado-trastorno de dolor como una dimensión de gravedad y su consiguiente eliminación del Eje I y reubicación en el Eje III, junto con otras condiciones médicas.

Además de estas críticas generales, los autores muestran los «defectos» de cada una de las subcategorias, haciendo especial hincapié en el TS, del que destacan cinco aspectos críticos: (a) Los pacientes con TS (según DSMIV) presentan síntomas significativos tanto físicos como psicológicos, por lo que apenas pueden ser considerados como representantes de una condición predominantemente somática.

(b) Existe un importante solapamiento entre el TS y trastornos de personalidad, en especial el límite.

(c) Aunque no es habitual realizar una evaluación detallada del número de síntomas para realizar el diagnóstico, hay evidencias de que el recuerdo de los síntomas por los pacientes es variable y que existe baja fiabilidad temporal en estudios longitudinales.

(d) La clasificación está basada meramente en un recuento de síntomas no explicados. Sin embargo, el TS se entiende mejor como un ex- 
tremo de gravedad de una dimensión de malestar, que como una categoría discreta.

(e) Realizar el diagnóstico no facilita al clínico disponer de una guía clara para abordar $\mathrm{y}$ tratar a estos pacientes.

\section{Réplicas a la propuesta abolicionista}

Las principales réplicas a las propuestas de Mayou y colaboradores fueron realizadas por Rief y su grupo de la Universidad de Marburg (Hiller y Rief, 2005; Rief, Henningsen y Hiller, 2006; Rief y Isaac, 2007), quienes han venido desarrollando un trabajo de investigación muy significativo en diversos aspectos relacionados con el concepto de somatización. Consideran que los pacientes con quejas somáticas no explicadas médicamente son el principal grupo de pacientes que evidencian un uso inadecuado de los servicios de salud, con más días de enfermedad y jubilación anticipada, por lo que es necesaria una clarificación de su diagnóstico. En primer lugar, consideran que el TS, lejos de ser una categoría «especulativa», está basado en una tradición de investigación rigurosa y creen que ha sido un avance importante respecto a diagnósticos y categorías previas al DSM-III (p.ej., histeria, neurastenia, sindromes funcionales) que no solo eran estigmatizantes, sino también poco fiables y no fundamentados en investigación.

En su opinión, en contraste con Mayou y colaboradores, a partir del DSM-III (y gracias al desarrollo de éste) se ha generado un importante cuerpo de investigación sobre estos trastornos $\mathrm{y}$ consideran que el avance en el tratamiento y diagnóstico de esos pacientes ha sido significativo, y que con su abolición existe el riesgo de regresar al conocimiento de esa área previo al DSM-III. Además, esa investigación y propuestas de tratamiento han sido generadas principalmente por investigadores procedentes de la salud mental, por lo que se correría el riesgo de detenerla si dejan de ser clasificados en el Eje I. Por otra parte, consideran que no existen motivos para esperar que los médicos especialistas no psiquiatras se preocupen más por los trastornos somatoformes, si estos pasan a clasificarse en el Eje III. Por otra parte, el hecho de que los tratamientos más efectivos sean de tipo psicosocial apoya su ubicación en el Eje I. Además argumentan que la mayoría de los pacientes con otros trastornos mentales (p.ej., ansiedad o depresión) también buscan ayuda principalmente en los médicos de atención primaria, en lugar de en profesionales de salud mental, y la búsqueda de ayuda la realizan habitualmente a través de quejas de síntomas físicos y no por manifestación de problemas «mentales», por lo que este no puede ser un motivo que justifique la reubicación del TS en el eje III (Rief et al. 2006).

Siguiendo a Starcevic (2006), consideran que la aceptación o no aceptación del paciente del diagnóstico, no puede ser un criterio para decidir dónde ha de ubicarse la clasificación de éste. Es improbable que el problema de la estigmatización se resuelva simplemente trasladando los diagnósticos al eje III. La no aceptación del diagnóstico no es cuestión del término que se utilice. La aceptación depende de la explicación que se proporciona, y no de que el diagnóstico de los síntomas «funcionales» sea clasificado en el Eje I, o en el Eje III (Rief et al., 2006). La aceptación mejorará con el incremento del conocimiento respecto al problema y el desarrollo de guías sobre cómo explicar el diagnóstico y cómo manejar a esos pacientes.

Sin embargo, están de acuerdo con Mayou y colaboradores en que la clasificación actual debe ser mejorada de modo sustancial a partir de los hallazgos clínicos y epidemiológicos, pero consideran que abolir o separar las categorías sería un paso atrás del DSM. Las críticas y comentarios de Rief y colaboradores a la clasificación actual pueden resumirse en los siguientes aspectos:

(a) El umbral del trastorno de somatización es excesivamente alto y el trastorno somatoforme indiferenciado está pobremente definido, por lo que los criterios actuales son poco útiles en la práctica. Los puntos de corte y listas de síntomas deben ser validados empíricamente.

(b) Es necesario incluir características psicológicas que sean específicas y relevantes en el mantenimiento y desarrollo de los síntomas.

(c) La clasificación basada en el recuento de síntomas ha sido un concepto muy criticado, pero consideran que el número de síntomas 
también es una característica importante para predecir el curso y pronóstico.

(d) La investigación actual ha puesto de manifiesto que las diferentes clasificaciones propuestas para los cuadros clínicos caracterizados por síntomas somáticos no explicados muestran un solapamiento significativo, y que las nuevas propuestas no llevan a resultados muy diferentes a los ya existentes. Además, hay una importante evidencia que indica que los distintos cuadros clínicos de trastornos «funcionales» incluidos en la CIE-10 (p.ej., dispepsia, colon irritable, dolores de cabeza) describen a los mismos tipos de pacientes que los se presentan con quejas múltiples (somatización). Por tanto, podría ser útil incluir estos trastornos (p.ej., fatiga crónica, colon irritable, dolor crónico) dentro de la categoría general que englobe a los pacientes con síntomas somáticos no explicados. Una nueva clasificación debe llevar a una reducción del número de subdiagnósticos y síndromes relacionados.

(e) Las clasificaciones basadas en cuadros monosintomáticos o relacionados con un único sistema orgánico, resultan poco fiables porque muchos de estos pacientes acaban padeciendo síntomas de otros sistemas.

(f) La utilización que los médicos hacen de los índices de síntomas no explicados médicamente está repleto de errores, ya que no resultan fiables para identificar a los pacientes que reciben esta etiqueta. Los nuevos criterios deben ser más fiables y válidos de modo que resulten útiles para diferenciar aquellos pacientes que presenten algún síntoma de los que puedan ser etiquetados como patológicos.

Consecuencias del debate: nuevas propuestas de clasificación

Como consecuencia del intenso debate y la necesidad de una modificación radical de la categoría para el DSM-V, a mediados de la década pasada se crearon dos foros de trabajo y discusión de gran envergadura científica: The conceptual issues in somatoform and similar disorders CISSD Project (Kroenke Y Mayou 2006), y el encuentro de expertos internacional en Presentación somática y trastornos psiquiá- tricos, organizado conjuntamente por la APA y la OMS (Regier, 2007). Junto con estos encuentros y grupos de trabajo otros autores relevantes han presentado sus propuestas de clasificación (p.ej., Rief y Hiller, 2005; Rief y Isaac, 2007; Fava y Wise, 2007; Kroenke, 2006; Martin, 1999; Fink et al., 2005; Noyes et al., 2008; Starcevic, 2006). Presentamos de forma resumida los principales puntos de acuerdo y divergencias entre estas diferentes propuestas (ver Tabla 4).

Aunque el grado de especificidad y alcance varía significativamente de unas a otras, las propuestas oscilan en una serie de ejes concretos que se resumen en la Tabla 4. En primer lugar, destaca la falta de consenso a la hora de determinar si los trastornos somatoformes quedan mejor clasificados como trastornos somáticos o como trastornos mentales. El mejor reflejo de este dilema es la falta de acuerdo al respecto en las recomendaciones propuestas por el CISSD Project (Kroenke et al., 2007). Sin embargo, podemos considerar mayoritarias las propuestas que consideran el Eje I como la mejor ubicación, siendo principalmente los autores firmantes del trabajo inicial de Mayou et al. (2005) los defensores de su eliminación como trastorno mental. Aparte de estas alternativas, algunos autores proponen la creación de un nuevo eje específico para estos cuadros clínicos (Starcevic, 2006; Fava y Wise, 2007). Concretamente Starcevic (2006) sugiere que este nuevo eje o categoría de trastornos entre lo somático y psíquico podría denominarse «síndromes por malestar psicosomático», idea planteada también por Rief y Sharpe (2004). Por su parte, Fava y Wise (2007) proponen agrupar los trastornos bajo la denominación de «factores psicológicos que afectan a una condición médica».

Respecto a la denominación de la categoría, existen fundamentalmente dos propuestas, muy vinculadas con la conceptuación del trastorno como mental o como somático. En primer lugar, los autores que abogan por abolir la categoría plantean la eliminación de los términos somatoforme y somatización (Mayou et al., 2005), proponiendo en su lugar la denominación de sindromes somáticos funcionales. Por otra parte, la mayoría de los autores que consideran que los trastornos deben seguir siendo clasificados como mentales, mantienen la denominación de 


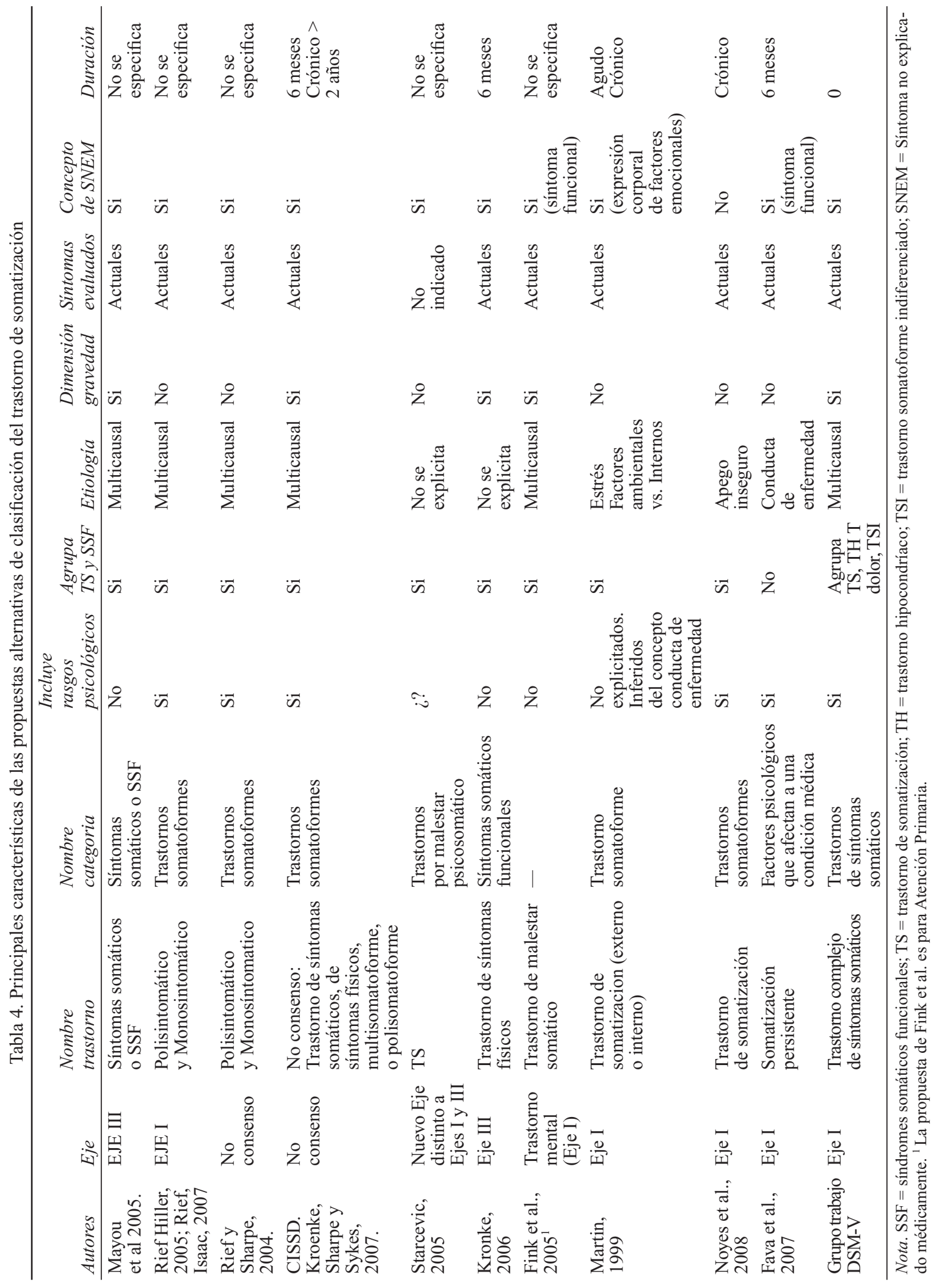


trastornos somatoformes (p.ej., Hiller y Rief, 2005; Noyes et al., 2008). En lo que sí existe consenso casi unánime es en lo inadecuado de la denominación de TS. Como denominación alternativa se proponen varios términos: trastorno de malestar somático, de síntomas somáticos, de síntomas físicos, multisomatoforme, o polisomatoforme.

Otro tema sobre el que parece existir consenso, que además modifica sustancialmente los criterios del DSM, es la inclusión de características psicológicas y/o conductuales en los criterios diagnósticos. Algunos de estos criterios psicológicos planteados son: intolerancia a sensaciones físicas, preocupación por los síntomas, atención selectiva, múltiples consultas médicas, tendencia a atribuir los síntomas a un trastorno médico general no diagnosticado, etc.

Un segundo elemento sobre el que hay consenso y que, desde nuestro punto de vista, puede tener consecuencias significativas terminológicas, nosológicas, clínicas y terapéuticas en el futuro, se refiere a la clasificación de forma conjunta de los trastornos somatoformes - actualmente en el Eje I- y de los trastornos/síndromes somáticos funcionales - actualmente en el Eje III. Lo que implica fundir bajo una misma categoría los cuadros somatoformes y los «psicosomáticos», claramente diferenciados en décadas precedentes (p.ej., Kellner, 1991; Levenson, 2007). Esta propuesta está fundamentada en los trabajos que muestran que las diferentes propuestas de clasificación de síndromes somáticos funcionales se solapan de forma manifiesta (Wessely, Nimnuam y Sharpe, 1999), en que comparten elementos comunes etiologicos y de tratamiento (p. ej., Alvaro y Traver, 2010; Rodero, García-Campayo, Casanueva y Buriel, 2009; Woodfolk y Allen, 2007) y que las nuevas clasificaciones de estos cuadros no dan lugar a resultados muy diferentes a las ya existentes (Dickinson, et al., 2003).

Varios autores consideran que evaluar el trastorno como un continuo de gravedad resulta especialmente pertinente en estos pacientes (Fink et al., 2005; Kroenke, 2006; Mayou et al., 2005), lo que indica que se defiende una aproximación más dimensional que categorial (Katon et al., 1991). Este aspecto está presente en mu- chas de las propuestas que se están realizando para el futuro DSM V en diferentes categorías diagnósticas, siendo el ejemplo más notable, sin duda, la referida a los trastornos de la personalidad. La propuesta de evaluación más detallada de la somatización como una dimensión de gravedad es la que ofrece Kroenke (2006), quien operativiza la gravedad a través del resultado en la escala PHQ-15 (Kroenke et al., 2002).

Hay diferentes elementos relacionados con la evaluación de los síntomas que son objeto de controversia. El primero se refiere al concepto de síntoma no explicado médicamente que, a pesar de ser criticado y puesto en cuestión por todos los autores, ha sido mantenido en todas las propuestas como «corazón» de la clasificación, con excepción de Noyes et al. (2008). Según este grupo, para realizar el diagnóstico de TS no es necesario que los síntomas origen del malestar deban ser etiquetados como no explicados médicamente. Un aspecto que unánimemente ha sido considerado en todas las propuestas es el de que, para realizar el diagnóstico, los síntomas evaluados sean los presentados en la actualidad y no los referidos a lo largo de la vida del paciente, como plantean los criterios actuales del DSM-IV. Este criterio se ve avalado por los estudios que indican problemas de fiabilidad temporal en la evaluación de los síntomas somáticos experimentados a lo largo de la vida (Leiknes, Finset, Moum y Sandanger, 2008; Simon y Gureje, 1999).

Por último, respecto al criterio relacionado con la presentación de un número determinado de síntomas, y de los sistemas orgánicos que deben estar afectados, a diferencia del DSM-IV o CIE-10 la mayoría de las propuestas omiten ofrecer criterios específicos al respecto. Únicamente Rief y Isaac (2007) distinguen entre polisintomáticos o monosintomáticos, y Fink et al. (2005) sugieren que podrían diagnosticarse subtipos en función de si predominan síntomas de un sistema específico (p.ej., subtipo gastrointestinal).A modo de epílogo en el siguiente epígrafe presentamos la propuesta provisional de nuevos criterios diagnósticos para el trastorno de somatización en el DSM-V (Dimsdale y Creed, 2009), que en gran medida, se puede considerar como un reflejo tanto de 
los acuerdos como de las divergencias entre los expertos.

\section{GRUPO DE TRABAJO DEL DSM-V: TRASTORNOS DE SÍNTOMAS SOMÁTICOS}

El grupo de trabajo para la revisión de los trastornos somatoformes se constituyó en febrero de 2008 (APA, www.psych.org.dsmv) con la denominación de grupo de trabajo de trastornos de estrés somático y que ha pasado a llamarse de trastornos de síntomas somáticos (Dimsdale y Creed, 2009). Ésta es también la denominación que se propone para que sustituya en el DSM-V la anterior categoría de trastornos somatoformes. En la primera publicación de este grupo de trabajo (Dimsdale y Creed, 2009) se plantean una serie de cuestiones sobre cuya base justifican la propuesta provisional de los nuevos criterios diagnósticos.

En primer lugar, se hacen eco de las múltiples críticas que ha recibido la categoría de trastornos somatoformes, afirmando que es «inaceptable para los pacientes e incomprensible para los médicos de atención primaria, que son quienes principalmente atienden a estos pacientes» (p. 473). Plantean también que el concepto de «no explicado médicamente» es especialmente problemático, porque perpetúa el dualismo mente-cuerpo, fundamenta el diagnóstico en un criterio negativo, y en muchas ocasiones los médicos no están de acuerdo en si un síntoma en particular es o no explicable médicamente. Por tanto, proponen que el diagnóstico se realice sobre la base de "criterios positivos», tomando como referencia las características conductuales y psicológicas del trastorno.Por otra parte señalan que, desde su punto de vista, no queda claro si la hipocondría y la somatización son verdaderamente trastornos distintos. Según estos autores, varios estudios han mostrado la existencia de un solapamiento significativo entre ambos trastornos (basándose en la revisión de Creed y Barsky, 2004). Consideran que las investigaciones futuras pueden verse dificultadas, más que favorecidas, al considerar de forma separada estos trastornos «cuando su independencia es incierta» (p. 473).
Propuesta de categoría: Trastornos de síntomas somáticos

Se trata de una categoría que agruparía a un conjunto de trastornos en los que predomina la presencia de síntomas somáticos que ocasionan malestar y disfunción psicosocial. Consideran que estos trastornos se presentan habitualmente en consultas no psiquiátricas ni psicológicas y que los factores psicológicos pueden iniciar, exacerbar o mantener los síntomas. Cuando se cumplan criterios también para otro trastorno mental como, por ejemplo, depresión mayor, han de realizarse ambos diagnósticos. La sección incluye cuatro trastornos que agrupa los actuales trastornos somatoformes y los facticios: trastorno complejo de síntomas somáticos (agudo o crónico), facticio (y facticio por poderes), de conversión, y síntomas somáticos no especificados.

Propuesta de diagnóstico: Trastorno complejo de síntomas somáticos

La categoría de trastorno complejo de síntomas somáticos incluye las anteriores clasificaciones de trastorno de somatización, somatoforme indiferenciado, de dolor, e hipocondría. Los criterios plantean realizar el diagnóstico a partir de la identificación de características cognitivas y conductuales «positivas», en contraposición a los anteriores diagnósticos de trastorno de somatización que implicaban, como único criterio, un diagnóstico «negativo», es decir, por exclusión de que exista explicación médica para los síntomas somáticos.

El diagnóstico incluye tres características principales: (1) la presencia de síntomas somáticos; (2) atribuciones erróneas, excesiva preocupación por los síntomas o por una enfermedad; y (3) incremento en el uso de recursos sanitarios. Los criterios propuestos de forma provisional se presentan en la Tabla 5. Esta propuesta ha sido sometida a discusión pública en febrero de 2010 en www.dsm5.org, y el primer estudio sistemático que ha valorado la utilidad clínica de estos criterios (Rief, Mewes, Martin Glaesmer y Brähler, 2011) concluye que, aunque es un propuesta válida para identificar per- 
sonas con elevada discapacidad y necesidad de tratamiento, y los criterios son estables en el tiempo, estos criterios siguen siendo muy restrictivos. Como nota final se incluye además la propuesta de considerar la hipocondría, tal y como se caracteriza en el DSM-IV, como una entidad heterogénea y ubicua, de tal modo que dependiendo de su presentación clínica en cada individuo particular, pueda considerarse como un trastorno por síntomas somáticos (y por tanto, incluido en este grupo) o como un trastorno de ansiedad. Se indica además que este planteamiento está siendo analizado también por los grupos de trabajo de trastornos de ansiedad, espectro obsesivo-compulsivo, disociativos, y por estrés.

Tabla 5. Propuesta de criterios diagnósticos para el trastorno complejo de síntomas somáticos

Para el diagnóstico, son necesarios los criterios A, B y C:

A. Sintomas somáticos.

Síntomas somáticos múltiples que ocasionan malestar, o un síntoma grave.

B. Atribuciones erróneas, excesiva preocupación [concern] o preocupación [preoccupation] por los síntomas o por la enfermedad. Se requiere al menos uno de los siguientes:

1. Elevada ansiedad por la salud.

2. Las sensaciones físicas normales son interpretadas como amenazantes y dañinas.

3. Tendencia a asumir lo peor respecto a la salud (catastrofización).

4. Creencia respecto a la gravedad de los síntomas físicos, a pesar de la evidencia contraria.

5. La preocupación por la salud ocupa un papel central en su vida.

C. Cronicidad. Aunque algunos de los síntomas pueden no estar siempre presentes, el estado de encontrarse sintomático es crónico y persistente.

Pueden aplicarse al diagnóstico las siguientes especificaciones opcionales cuando en la presentación clínica predomina una de las siguientes:

XXX.1 Multiplicidad de síntomas somáticos(antes TS)

XXX.2 Elevada ansiedad por la salud (antes Hipocondría):Si los pacientes presentan únicamente ansiedad por la salud en ausencia de síntomas somáticos, puede ser más apropiado que sea diagnosticado como trastorno de ansiedad.

XXX.3 Trastorno de dolor. Esta clasificación está reservada para personas que presentan predominantemente quejas de dolor, y que también presentan muchas de las características descritas en el criterio B. Pacientes con otras presentaciones de dolor pueden adaptarse mejor a otros diagnósticos psiquiátricos, como depresión mayor o trastorno de adaptación.

Para evaluar la gravedad del trastorno, están disponibles medidas para la gravedad de síntomas somáticos (véase por ejemplo PHQ, Kroenke et al., 2002). También hay escalas disponibles para evaluar la gravedad de las atribuciones erróneas del paciente y sus preocupaciones excesivas (véase por ejemplo el inventario de Whiteley, Pilowsky, 1967).

Nota. Fuente: http://www.dsm5.org.

\section{DISCUSIÓN Y CONCLUSIONES}

La «creación» del TS como entidad diagnóstica es un ejemplo paradigmático de operativización de un problema que se viene observando en la clínica desde hace siglos. Desde esta perspectiva, el planteamiento que hizo el DSM-III sobre este trastorno ha de considerarse como positivo. No obstante, la evolución y utilidad clínica de esa propuesta clasificatoria se ha mostrado inadecua- da, y como consecuencia, su uso no se ha consolidado en la práctica y la investigación basada en los criterios diagnósticos específicos que se propusieron ha proporcionado magros resultados. Sin embargo, y a pesar de todo, también hay que reconocer que la propuesta del DSM-III supuso poner en evidencia la necesidad de investigar el fenómeno de la somatización y, en este sentido, ha servido para aumentar nuestro conocimiento sobre este complejo y esquivo problema. 
Por lo que se refiere a su epidemiología, la principal conclusión que podemos obtener es la de que aunque la prevalencia del TS es baja, no es despreciable. Y, si en lugar de los criterios DSM se utilizan los de la CIE-10, puede ser considerablemente elevada. Ahora bien: cuando se utilizan alternativas menos restrictivas que las que propone el DSM para el diagnóstico del $\mathrm{TS}$, las tasas de prevalencia aumentan de forma espectacular tanto en el contexto de la atención primaria de salud, como en la población general sana: en torno al $20 \%$. La pregunta que inevitablemente surge es si verdaderamente esas tasas responden a la realidad. La respuesta es, desde nuestro punto de vista, una vez analizados los estudios en los que se basan tales datos, negativa. La mera constatación de la experiencia de síntomas somáticos carentes de explicación médica durante un período amplio de tiempo, no justifica per se un diagnóstico de trastorno mental. En consecuencia, resulta evidente la necesidad de considerar otros aspectos, tanto psicológicos como socio-ambientales y biológicos, para llegar a la conclusión de que una persona padece un trastorno de estas características y requiere, en consecuencia, un tratamiento específico.

La investigación psicológica sobre el TS viene proporcionando información relevante sobre el tipo de variables que deberían ser tenidas en consideración para caracterizar adecuadamente el TS. De la somera revisión que hemos presentado, pueden extraerse algunas conclusiones importantes. En primer lugar, que existe una relación moderada, aunque clínicamente significativa, entre hipocondría y somatización, y que los pacientes hipocondríacos presentan un mayor número de síntomas somáticos que los no hipocondríacos. Respecto a la co-ocurrencia entre ambos trastornos, aunque los informes más recientes indican que es elevada, nuestra impresión, que no coincide con la del grupo de trabajo del DSM-V, es que los estudios reflejan que se trata de trastornos independientes cuando se utilizan criterios completos del TS y no reducciones más o menos afortunadas del TS a un número muy limitado de síntomas. De hecho, lo que los datos indican es que, por un lado, los sujetos con diagnóstico de TS, y en menor medida, aunque también, los sujetos con «somatización abreviada», presentan una comorbilidad baja con hipocondría. Pero, por otro, sucede lo contrario si el problema se examina desde la hipocondría, ya que los pacientes diagnosticados de hipocondría presentan una comorbilidad elevada con somatización si se utilizan los criterios de somatización abreviada, si bien la comorbilidad disminuye si se aplican los criterios estrictos del trastorno de somatización. En este sentido, es importante recordar que la presencia de síntomas somáticos en pacientes hipocondríacos forma parte de los criterios diagnósticos del trastorno al indicar que la «preocupación y miedo a tener, o la convicción de padecer, una enfermedad grave» se realiza «a partir de la interpretación de síntomas físicos» (APA, 2000). Obviamente, si el clinico aplica en sentido estricto este criterio, debe asegurarse antes que nada de que el paciente experimenta síntomas somáticos, pues de lo contrario no cabe hablar de interpretación alguna de ellos. Además, parece esperable que un diagnóstico como el de somatización abreviada que se da en un $20 \%$ de la población en muestras de atención primaria (Creed y Barsky, 2004), aparezca de forma muy frecuente en pacientes hipocondríacos.

Un segundo elemento especialmente importante es el que se refiere a las explicaciones causales, las atribuciones, que dan los pacientes con TS a sus síntomas somáticos. Los datos indican que hay al menos tres factores atribucionales diferentes, en función de la población estudiada: mientras que las personas de población general realizan preferentemente atribuciones normales, los pacientes con trastornos emocionales hacen preferentemente atribuciones psicológicas y son los pacientes con elevada preocupación por la salud los que realizan más frecuentemente atribuciones somáticas. Este modelo de tres factores ha sido confirmado en un reciente estudio realizado en el contexto de la atención sanitaria pública en salud mental (López-Santiago, 2011).

En tercer lugar se encuentran los estudios que examinan la relación entre somatización y dimensiones de rasgos como la alexitimia y el neuroticismo, así como con constructos más específicos del ámbito de la somatización. La amplificación somatosensorial se encuadra en 
este último grupo y ha recibido una gran atención desde que Barsky la hipotetizó como posible causa de somatización. La mayoría de los estudios constatan que existen relaciones entre la SSAS y diversas medidas de somatización, pero al mismo tiempo muestran que no es específica y resulta difícil poder extraer conclusiones claras respecto al significado de esa relación. En cuanto a la alexitimia, los estudios son consistentes en establecer una relación entre somatización y la subescala dificultad para identificar sentimientos y en menor medida con la de dificultad para expresar sentimientos, mientras que la tercera subescala (pensamiento orientado a detalles externos) no parece asociarse con la somatización. Por otra parte, de nuevo parece que esa relación no es específica ya que no están claras las diferencias con otros grupos de pacientes caracterizados por la presencia de síntomas. Finalmente los estudios que han examinado la asociación entre somatización y neuroticismo tampoco han proporcionado resultados que permitan hablar de una especificidad, lo que por otro lado era esperable dado el rol que expertos muy relevantes otorgan a esta dimensión de personalidad como transversal y común al vasto campo de los trastornos emocionales entre los que, sin duda, se incluye la somatización (p.ej., Brown y Barlow, 2009).

La relación del TS con trastornos de ansiedad y muy especialmente, con los depresivos, es uno de los resultados que de forma más consistente han mostrado los estudios sobre el trastorno de somatización realizados en los últimos 30 años. Sin embargo, a pesar del elevado solapamiento entre somatización y depresión, existe consenso para considerar ambos trastornos como independientes y para no cuestionar la validez convergente (especificidad respecto a otros) del trastorno de somatización (Dimsdale y Creed, 2009; Lowe, Mundt et al., 2008). Los principales argumentos esgrimidos para considerarlos como entidades clínicas independientes han sido tres. En primer lugar, el grupo de trabajo para el DSM-V justifica su independencia en que aproximadamente la mitad de los somatizadores no presentan comorbilidad con ansiedad ni con depresión, a nivel de trastornos completos (Dimsdale y Creed, 2009). En segundo lugar, un meta-análisis de la relación entre síntomas no explicados médicamente, ansiedad, y depresión (Henningsen et al., 2003) concluye que los trastornos funcionales no son explicados completamente por los síntomas de ansiedad o depresión comórbidos. Finalmente, el coste sanitario de los pacientes somatizadores continúa siendo muy elevado, aun controlando el efecto de los síntomas de ansiedad y depresión (Barsky, Orav y Bates, 2005).

El reconocimiento de la necesidad de realizar una delimitación más precisa del fenómeno de la somatización o del TS propiamente dicho, en su caso, ha llevado a los expertos a multiplicar esfuerzos para alcanzar un consenso en este aspecto crucial de la delimitación diagnóstica. Este es el primer paso necesario para poder avanzar en el conocimiento del problema y mejorar la situación de los afectados. El análisis de las diferentes propuestas clasificatorias que se vienen presentando en los últimos años mantienen algunos puntos de acuerdo casi unánime: eliminación del término somatización, inclusión de rasgos psicológicos/conductuales en los criterios diagnósticos, agrupación de trastornos somatoformes y funcionales, o valoración únicamente de los síntomas actuales. Pero también existen divergencias significativas. De éstas, algunas tienen una base fundamentalmente teórica o conceptual: por ejemplo, cuál es la terminología más acertada, cuál el eje más adecuado para la clasificación, o el problema de mantener una clasificación basada en una filosofía dualista. Otras pueden tener una respuesta empírica a partir de los datos que puede ofrecer la investigación: por ejemplo, la validez de las distintas clasificaciones, los aspectos psicológicos que pueden asociarse a los cuadros caracterizados por somatizaciones, o la utilidad de incluir síntomas específicos en la nosografía.

La nueva propuesta clasificatoria para el DSM-V recoge algunas de las evidencias de la investigación precedente, como la inclusión de pacientes mono y multisintomáticos en el mismo diagnóstico, la eliminación de un listado específico de síntomas dada la falta de estabilidad temporal de ese criterio y la inclusión de algunos rasgos psicológicos en sus criterios diagnósticos, huyendo de confiar exclusivamente en un listado de síntomas. Sin embargo, nos parece arbitraria la inclusión, dentro de los mis- 
mos criterios, de pacientes actualmente diagnosticados como TS e hipocondría. Como hemos visto, los estudios publicados sobre la comorbilidad de ambos trastornos apuntan precisamente en la dirección contraria. Y, según nuestra propia experiencia clínica y de investigación, las características de ambos grupos de pacientes son claramente distintas (López-Santiago, 2011). Además, este aspecto ha sido el más cuestionado en las primeras publicaciones que han valorado la nueva propuesta de clasificación (Rief, 2010; Schröder y Fink; 2010). Consideramos también que puede ser contraproducente y estigmatizante, además de tener escasa utilidad clínica y suponer un grave error epistemológico y fenomenológico, que dentro de la misma categoría se incluya el trastorno facticio. Si esta idea se mantiene, se potenciará la equiparación, en lugar de su diferenciación, entre síntoma no explicado médicamente y síntoma fingido, uno de uno de los caballos de batalla más importantes de los cuadros somatoformes.

Para concluir, resulta evidente que estamos asistiendo solo al inicio de un cambio importante en la conceptuación del TS, que se refleja no solo en las nuevas propuestas diagnósticas, de futuro todavía incierto, sino sobre todo en la forma de abordar su estudio y comprensión. Las decisiones respecto a nuevos criterios de clasificación, aunque tengan que ser sometidos a consenso, han de fundamentarse en un análisis adecuado de la investigación existente, lo que conlleva que de ningún modo debieran prevalecer posicionamientos o intereses particulares frente a los datos de la investigación, obviamente siempre revisables y sujetos a interpretación. Todavía queda un largo camino por recorrer para clarificar la naturaleza y clasificación de los cuadros actualmente diagnosticados como TS: su avance dependerá en gran medida del acierto, o sea de la fundamentación científica, en que se base su definición operativa.

\section{REFERENCIAS}

Allen, L. A., Gara, M. A., Escobar, J. I., W aitzkin, H., \& Silver, R. C. (2001). Somatization: A debilitating syndrome in primary care. Psychosomatics, 42, 63-67.
Alvaro, T. y Traver, F. (2010). Una visión psiconeuroinmunológica de la fibromialigia. Revista de Psicopatología y Psicología Clínica, 15, 149-163.

American Psychiatric Association (APA, 1980). Diagnostic and statistical manual of mental disorders (3rd ed.) (DSM-III). Washington, DC: APA.

American Psychiatric Association (APA) (1987). Diagnostic and statistical manual of mental disorders (3rd ed. rev.) (DSM-III-R). Washington, DC: APA.

American Psychiatric Association (APA) (1994). Diagnostic and statistical manual of mental disorders (4th ed.) (DSM-IV). Washington, DC: APA.

American Psychiatric Association (APA) (2000). Diagnostic and statistical manual of mental disorder (4th ed.), Text revision (DSM-IV-TR). Washington, DC: APA.

Bach, M., \& Bach, D. (1996). Alexithymia in somatoform disorder and somatic disease: A comparative study. Psychotherapy and Psychosomatics, 65, 150-152.

Bagby, R. M., Parker, J. D., \& Taylor, G. J. (1994). The twenty-item Toronto Alexithymia Scale-I. Item selection and cross-validation of the factor structure. Journal of Psychosomatic Research, 38, 2332

Barsky, A. J., Goodson, J. D., Lane, R. S., \& Cleary, P. D. (1988). The amplification of somatic symptoms. Psychosomatic Medicine, 50, 510-519.

Barsky, A. J., Orav, E. J., \& Bates, D. W. (2005). Somatization increases medical utilization and costs independent of psychiatric and medical comorbidity. Archives of General Psychiatry, 62, 903-910.

Barsky, A. J., Wyshak, G., \& Klerman, G. (1990). The somatosensory amplification scale and its relationship to hypochondriasis. Journal of Psychiatric Research, 24, 323-334.

Barsky, A. J., \& Wyshak, G. (1990). Hypochondriasis and somatosensory amplification. British Journal of Psychiatry, 157, 404-409.

Belloch, A., Ayllón, E., Martínez, M. P., Castañeiras, C., y Jiménez, M. A. (1999). Sensibilidad a las sensaciones corporales e hipocondría. Revista de Psicopatología y Psicología Clínica, 4, 1-14.

Brown, F. W., Golding, J. M., \& Smith, G. R. (1990). Psychiatric comorbidity in primary care somatization disorder. Psychosomatic Medicine, 52, 445-451.

Brown, T. A., \& Barlow, D. H. (2009). A proposal for a dimensional classification system based on the shared features of the DSM-IV anxiety and mood disorders: Implications for assessment and treatment. Psychological Assessment, 21, 256-271.

Castañeiras, C. (2001). Avances en psicopatología y clínica de la hipocondría. Tesis doctoral. Universidad de Valencia.

Cloninger, C. R., Reich, T., \& Guze, S. B. (1975). The multifactorial model of disease transmission: III. Familial relationship between sociopathy and hysteria 
(Briquet's syndrome). British Journal of Psychiatry, 127, 23-32.

Creed, F., \& Barsky, A. (2004). A systematic review of the epidemiology of somatisation disorder and hypochondriasis. Journal of Psychosomatic Research, 56, 391408.

De Gucht, V. (2003). Stability of neuroticism and alexithymia in somatization. Comprehensive Psychiatry, 44, 466-471.

De Waal, M. W., Arnold, I. A., Eekhof, J. A., Assendelft, W. J., \& van Hemert, A. M. (2008). Follow-up study on health care use of patients with somatoform, anxiety and depressive disorders in primary care. $\mathrm{BMC} \mathrm{Fa-}$ mily Practice, 9, 5.

Deary, V., Chalder, T., \& Sharpe, M. (2007). The cognitive behavioural model of medically unexplained symptoms: A theoretical and empirical review. Clinical Psychology Review, 27, 781-797.

Dickinson, W. P., Dickinson, L. M., deGruy, F. V., Candib, L. M., Main, D. S., Libby, A. M., et al. (2003). The somatization in primary care study: A tale of three diagnoses. General Hospital Psychiatry, 25, 1-7.

Dimsdale, J., \& Creed, F. (2009). The proposed diagnosis of somatic symptom disorders in DSM-V to replace somatoform disorders in DSM-IV. A preliminary report. Journal of Psychosomatic Research, 66, 473-476.

Duddu, V., Isaac, M. K., \& Chaturvedi, S. K. (2003). Alexithymia in somatoform and depressive disorders. Journal of Psychosomatic Research, 54, 435-438.

Duddu, V., Isaac, M. K., \& Chaturvedi, S. K. (2006). Somatization, somatosensory amplification, attribution styles and illness behaviour: A review. International Review of Psychiatry, 18, 25-33.

Escobar, J. I., Gara, M., Silver, R. C., Waitzkin, H., Holman, A., \& Compton, W. (1998). Somatisation disorder in primary care. British Journal of Psychiatry, 173, 262-266.

Escobar, J. I., Golding, J. M., Hough, R. L., Karno, M., Burnam, M. A., \& Wells, K. B. (1987). Somatization in the community: relationship to disability and use of services. American Journal of Public Health, 77, 837840.

Escobar, J. I., Rubio-Stipec, M., \& Canino, G. (1989). Somatic Symptoms Index (SSI): A new and abridged somatization construct: Prevalence and epidemiological correlates in two larges community samples. Journal of Nervous and Mental Disease, 177, 140146.

Escobar, J. I., Waitzkin, H., Silver, R. C., Gara, M., \& Holman, A. (1998). Abridged somatization: A study in primary care. Psychosomatic Medicine, 60, 466-472.

Escobar, J. I., \& Gureje, O. (2007). Influence of cultural and social factors on the epidemiology of idiopathic somatic complaints and syndromes. Psychosomatic Medicine, 69, 841-845.
Escobar, J. I., Burnam, M. A.; Karno, M; Forsythe, A., \& Golding, J. M. (1987). Somatization in the comunity. Archives of General Psychiatry, 44, 713-718.

Fabbri, S., Fava, G. A., Sirri, L., \& Wise, T. N. (2007). Development of a new assessment strategy in psychosomatic medicine: The diagnostic criteria for psychosomatic research. Advances in Psychosomatic Medicine, 28, 1-20.

Faravelli, C., Salvatori, F., Galassi, L., Aiazzi, L., Drei, C.,\& Cabras, P. (1997). Epidemiology of somatoform disorders: A community survey in Florence. Social Psychiatry and Psychiatric Epidemiology, 32, 24-29.

Fava G.A. \& Wise, T. N. (2007). Issues for DSM-V: psychological factors affecting either identified or feared medical conditions: A solution for somatoform disorders. American Journal of Psychiatry, 164, 1002-3.

Feighner, J. P., Robins, E., Guze, S. B., Woodruff, R. A., Jr., Winokur, G., \& Munoz, R. (1972). Diagnostic criteria for use in psychiatric research. Archives of General Psychiatry, 26, 57-63.

Fink, P., Rosendal, M., \& Olesen, F. (2005) Classification of somatization and functional somatic symptoms in primary care. Australian \& New Zeland Journal of Psychiatry, 39, 772-781.

Fink, P., Sorensen, L., Engberg, M., Holm, M., \& MunkJorgensen, P. (1999). Somatization in primary care. Prevalence, health care utilization, and general practitioner recognition. Psychosomatics, 40, 330-338.

Gureje, O., Simon, G. E., Ustun, T. B., \& Goldberg, D. P. (1997). Somatization in cross-cultural perspective: AWorld Health Organization study in primary care. American Journal of Psychiatry, 154, 989-995.

Gureje, O., \& Simon, G. E. (1999). The natural history of somatization in primary care. Psychological Medicine, 29, 669-676.

Guze, S. B. (1963). The clinical diagnosis of hysteria. Medical Times, 91, 1149-1152.

Guze, S. B. (1967). The diagnosis of hysteria: What are we trying to do? American Journal of Psychiatry, 124, 491-498.

Guze, S. B. (1970). Th role of follow-up studies: Their contribution to diagnostic classification as applied to hysteria. Seminars in Psychiatry, 2, 392-402.

Guze, S. B., Woodruff, R. A., Jr., \& Clayton, P. J. (1972). Sex, age, and the diagnosis of hysteria (Briquet's syndrome). American Journal of Psychiatry, 129, 745-748.

Hanel, G., Henningsen, P., Herzog, W., Sauer, N., Schaefert, R., Szecsenyi, J., et al. (2009). Depression, anxiety, and somatoform disorders: Vague or distinct categories in primary care? Results from a large cross-sectional study. Journal of Psychosomatic Research, 67, 189-197.

Henningsen, P., Jakobsen, T., Schiltenwolf, M.,\& Weiss, M. G. (2005). Somatization revisited: Diagnosis and 
perceived causes of common mental disorders. Journal of Nervous and Mental Disease, 193, 85-92.

Henningsen, P., Zimmermann, T., \& Sattel, H. (2003). Medically unexplained physical symptoms, anxiety, and depression: A meta-analytic review. Psychosomatic Medicine, 65, 528-533.

Hiller, W., Rief, W., \& Brahler, E. (2006). Somatization in the population: From mild bodily misperceptions to disabling symptoms. Social Psychiatry and Psychiatric Epidemiology, 41, 704-712.

Hiller, W., \& Rief, W. (2005). Why DSM-III was right to introduce the concept of somatoform disorders. Psychosomatics, 46, 105-108.

Katon, W., Kleinman, A., \& Rosen, G. (1982a). Depression and somatization: A review. Part I. American Journal of Medicine, 72, 127-135.

Katon, W., Kleinman, A., \& Rosen, G. (1982b). Depression and somatization: A review. Part II. American Journal of Medicine, 72, 241-247.

Katon, W., Lin, E., Von Korff, M., Russo, J., Lipscomb, P., $\&$ Bush, T. (1991). Somatization: a spectrum of severity. American Journal of Psychiatry, 148, 34-40.

Kellner, R. (1985). Functional somatic symptoms and hypochondriasis. A survey of empirical studies. Archives of General Psychiatry, 42, 821-833.

Kellner, R. (1986). Somatization and Hypochondriasis. New York: Preager.

Kellner, R. (1990). Somatization. Theories and research. Journal of Nervous and Mental Disease, 178, 150-160.

Kellner, R. (1991). Psychosomatic syndromes and somatic symptoms. Washington DC: American Psychiatric Press.

Kessler, D., Lloyd, K., Lewis, G., \& Gray, D. P. (1999). Cross sectional study of symptom attribution and recognition of depression and anxiety in primary care. British Medical Journal, 318, 436-439.

Kirmayer, L. J. \& Robbins, J. M. (1996). Patients who somatize in primary care: A longitudinal study of cognitive and social characteristics. Psychological Medicine, 26, 937-51.

Kirmayer, L. J., Groleau, D., Looper, K. J., \& Dao, M. D. (2004). Explaining medically unexplained symptoms. Canadian Journal of Psychiatry. Revue Canadienne de Psychiatrie, 49, 663-672.

Kirmayer, L. J., Robbins, J. M., \& Paris, J. (1994). Somatoform disorders: Personality and the social matrix of somatic distress. Journal of Abnormal Psychology, 103, 125-136.

Kirmayer, L. J.\& Taillefer S. (1997) Somatoform disorders. In Turner S. M., and Hersen, M. (Eds.). Adultspsychopatology and diagnosis. New York: Wiley ySons.

Kroenke, K. \& Sharpe M. (2006) Special Mini-Series on Somatoform Disorders. Journal of Psychosomatic Research, 60, 323.
Kroenke, K. (2003). Patients presenting with somatic complaints: Epidemiology, psychiatric comorbidity and management. International Journal of Methods in Psychiatry Research, 12, 34-43.

Kroenke, K. (2006). Physical symptom disorder: A simpler diagnostic category for somatization-spectrum conditions. Journal of Psychosomatic Research, 60, 335-339.

Kroenke, K. (2008). Trastornos somatomorfos y controversias diagnósticas. En J. Levenson, D. Gitlin, y C. Crone (Eds.), Clínicas psiquiátricas norteamericanas. Medicina Psicosomática. Barcelona: Masson.

Kroenke, K., Arrington, M. E., \& Mangelsdorff, A. D. (1990). The prevalence of symptoms in medical outpatients and the adequacy of therapy. Archives of Internal Medicine, 150, 1685-1689.

Kroenke, K., Sharpe, M., \& Sykes, R. (2007). Revising the classification of somatoform disorders: Key questions and preliminary recommendations. Psychosomatics, 48, 277-85.

Kroenke, K., Spitzer, R. L., deGruy, F. V., \& Swindle, R. (1998). A symptom checklist to screen for somatoform disorders in primary care. Psychosomatics, 39, 263-272.

Kroenke, K., Spitzer, R. L., \& Williams, J. B. (2002). The PHQ-15: Validity of a new measure for evaluating the severity of somatic symptoms. Psychosomatic Medicine, 64, 258-266.

Kupfer, D. J., First, M. B.,\& Regier, D. A. (2002). A Research Agenda for DSM- $V$. Washington, DC: American Psychiatric Press.

Leibbrand, R., Hiller, W., \& Fichter, M. M. (2000). Hypochondriasis and somatization: Two distinct aspects of somatoform disorders? Journal of Clinical Psychology, $56,63-72$.

Leiknes, K. A., Finset, A., Moum, T., \& Sandanger, I. (2008). Overlap, comorbidity, and stability of somatoform disorders and the use of current versus lifetime criteria. Psychosomatics, 49, 152-162.

Levenson, J. L. (2007). Tratado de Medicina Psicosomática. Barcelona: Ars Médica.

Lieb, R., Meinlschmidt, G., \& Araya, R. (2007). Epidemiology of the association between somatoform disorders and anxiety and depressive disorders: An update. Psychosomatic Medicine, 69, 860-863.

Lipowski, Z. J. (1990). Somatization and depression. Psychosomatics, 31, 13-21.

Lobo, A., García-Campayo, J., Campos, R., Marcos, G., Pérez-Echeverría, M., \& GWPZ (1996). Somatisation in primary care in Spain. I. Estimates of prevalence and clinical characteristics. British Journal of Psychiatry, 168, 344- 353.

López-Santiago, J. (2011). Delimitación diagnóstica y clínica del trastorno de somatización. Tesis doctoral. Servicio de Publaciones Universidad de Valencia.

López-Santiago, J. y Belloch, A. (2002). La somatización como síntoma y como síndrome: Una revisión del 
trastorno de somatización. Revista de Psicopatología y Psicología Clínica, 7, 73-93.

Lowe, B., Mundt, C., Herzog, W., Brunner, R., Backenstrass, M., Kronmuller, K., et al. (2008). Validity of current somatoform disorder diagnoses: Perspectives for classification in DSM-V and ICD-11. Psychopatho$\log y, 41,4-9$.

MacLeod, R. J., McNamee, J. E., Boyle, M. H., Offord, D. R., \& Friedrich, M. (1999). Identification of childhood psychiatric disorder by informant: Comparisons of clinic and community samples. Canadian Journal of Psychiatry, 44, 144-150.

Mayou, R., Kirmayer, L. J., Simon, G., Kroenke, K., \& Sharpe, M. (2005). Somatoform disorders: Time for a new approach in DSM-V. American Journal of Psychiatry, 162, 847-855.

Mayou, R., Levenson, J., \& Sharpe, M. (2003). Somatoform disorders in DSM-V. Psychosomatics, 44, 449-451.

Noyes, R. Jr, Stuart, S., Watson, D. B., \& Langbehn, D. R. (2006). Distinguishing between hypochondriasis and somatization disorder: A review of the existing literature. Psychotherapy and Psychosomatics, 75, 270-281.

Noyes, R. Jr., Stuart, S. P., \& Watson, D. B. (2008) A reconceptualization of the somatoform disorders. Psychosomatics., 49, 14-22.

Organización Mundial de la Salud (OMS) (1992). Trastornos mentales y del comportamiento, $10{ }^{a}{ }^{a}$ edición. (CIE-10). Madrid: Meditor.

Parker, G., \& Parker, K. (2003). Influence of symptom attribution on reporting depression and recourse to treatment. Australian and New Zealand Journal of Psychiatry, 37, 469-474.

Pedrosa Gil, F., Ridout, N., Kessler, H., Neuffer, M., Schoechlin, C., Traue, H. C., et al. (2008). Facial emotion recognition and alexithymia in adults with somatoform disorders. Depression and Anxiety, 25, 133-141.

Perley, M. J., \& Guze, S. B. (1962). Hysteria--the stability and usefulness of clinical criteria. A quantitative study based on a follow-up period of six to eight years in 39 patients. New England Journal of Medicine, 266, 421-426.

Regier, D. A. (2007). Somatic presentations of mental disorders: Refining the research agenda for DSM-V. Psychosomatic Medicine, 69, 827-828.

Rief, W. (2010). Painting the picture of distressing somatic symptoms. Journal of Psychosomatic Research, 68, 1-3.

Rief, W., Henningsen, P., \& Hiller, W. (2006). Classification of somatoform disorders. American Journal of Psychiatry, 163,746-47.

Rief, W., Hiller, W., yMargraf, J. (1998). Cognitive aspects of hypochondriasis and the somatization syndrome. Journal of Abnormal Psychology, 107, 587-595.
Rief, W., Mewes, R., Martin, A., Glaesmer, H.,\& Brähler, E. (2011). Evaluating new proposals for the psychiatric classification of patients with multiple somatic symptoms. Psychosomatic Medicine, 73, 760-768.

Rief, W.\& Isaac, M. (2007). Are somatoform disorders mental disorders? A contribution to current debate. Current Opinion in Psychiatry, 20, 143-146

Robbins, J. M., \& Kirmayer, L. J. (1991). Attributions of common somatic symptoms. Psychological Medicine, 21, 1029-1045.

Robins, L. N., \& Regier, D. A. (1991). Psychiatric disorders in America: the epidemiologic catchment area study. New York: Free Press.

Rodero, B., García-Campayo, J., Casanueva, B., y Buriel, Y. (2009). Tratamientos no farmacológicos en fibromialgia: Una revisión actual. Revista de Psicopatología y Psicología Clínica, 14, 137-151.

Sandín, B., Santed, M.A., Chorot, P., y Valiente, R. (1996). Alexitimia, afecto positivo y negativo y reactividad al estrés: Relaciones con sintomatología somática y enfermedad. Análisis y Modificación de Conducta, 22, 435-460.

Schappert, S. M. (1992). National Ambulatory Medical Care Survey: 1990 summary. Advance Data, 18, 1-11.

Schroder, A., \& Fink, P. (2010). The proposed diagnosis of somatic symptom disorders in DSM-V: Two steps forward and one step backward? Journal of Psychosomatic Research, 68, 95-96; author reply 99-100.

Sensky, T. (1994). Somatization: Syndromes or processes? Psychotherapy and Psychosomatics, 61, 1-3.

Sharpe, M., \& Mayou R. (2004). Somatoform disorders: A help or hindrance to good patient care? British Journal of Psychiatry, 184, 465-67.

Sifneos, P. E. (1973). The prevalence of 'alexithymic' characteristics in psychosomatic patients. Psychotherapy and Psychosomatics, 22, 255-262.

Simon, G. E., \& Gureje, O. (1999). Stability of somatization disorder and somatization symptoms among primary care patients. Archives of General Psychiatry, 56, 90-95.

Spitzer, R. L., Endicott, J., \& Robins, E. (1975). Research diagnostic criteria. Psychopharmacology Bulletin, 11, 22-25.

Spitzer, R. L., Endicott, J., \& Robins, E. (1978). Research diagnostic criteria: Rationale and reliability. Archives of General Psychiatry, 35, 773-782.

Starcevic, V. (2006). Somatoform disorders and DSM-V: Conceptual and political issues in the debate. Psychosomatics, 47, 277-281.

Swartz, M., Blazer, D., George, L., \& Landerman, R. (1986). Somatization disorder in a community population. American Journal of Psychiatry, 143, 1403-1408.

Taylor, G. J., Ryan, D., \& Bagby, R. M. (1985). Toward the development of a new self-report alexithymia scale. Psychotherapy and Psychosomatics, 44, 191-199. 
Toft, T., Fink, P., Oernboel, E., Christensen, K., Frostholm, L., \& Olesen, F. (2005). Mental disorders in primary care: Prevalence and co-morbidity among disorders. results from the functional illness in primary care (FIP) study. Psychological Medicine, 35, 1175-1184.

Wessely, S., Nimnuan, C., \& Sharpe, M. (1999). Functional somatic syndromes: One or many?Lancet, 354, 936-939.
Woodruff, R. A., Goodwin, D. W., \& Guze, S. B. (1978). El Diagnóstico en Psiquiatría. Mexico D. F.: El Manual Moderno. (Original: 1974).

Woolfolk, R. L., \& Allen, L. A. (2007). Treating Somatization: A Cognitive-Behavioral Approach. New York: Guilford Press. 